\title{
Effective Team Teaching between Local and Native-speaking English Teachers
}

\author{
David Carless \\ University of Hong Kong, China
}

\author{
Elizabeth Walker \\ Hong Kong Institute of Education, Hong Kong, China
}

This paper focuses on collaboration between native-speaking English teachers (NETs) and local English teachers (LETs) in Hong Kong secondary schools. It examines some of the strengths and weaknesses of NETs and LETs documented in the international literature. It reviews, in various contexts, schemes where team teaching has been carried out. Using case studies of selected effective practitioners augmented by recent published research, we discuss how native and non-native teachers worked together and how their collaboration impacted on themselves and their students. Our analysis elaborates on some inter- and intra-personal factors facilitating the team teaching, balanced by some of the dilemmas particularly with respect to educational philosophies. The paper concludes by arguing for relationships between particular features of the collaborations and theorised conditions for second language acquisition.

doi: $10.2167 / l e 627.0$

Keywords: collaboration, native-English teachers, non-native English teachers, team teaching

\section{Introduction}

This paper explores some of the potential of team teaching between nativespeaking ${ }^{1}$ English teachers (NETs) and local non-native-speaking English teachers (LETs) in Hong Kong secondary schools. Our analysis is drawn from two main sources, our own recent data collection, involving classroom observation and interviews in schools; and published literature, particularly a major report (Storey et al., 2001). We focus on three main themes: the nature of collaboration between NETs and LETs; impact on students; and impact on teachers (both LETs and NETs).

NET schemes in Hong Kong secondary ${ }^{2}$ schools have existed since 1987. The current scheme implemented since 1998 seeks to enable NETs to enhance the teaching of English by: acting as English language resource persons; assisting in school-based teacher development; and helping to foster an enabling environment for students to practise their oral English skills (Storey et al., 2001).

The respective capacities of NETs and LETs have been discussed in the literature, especially through Medgyes' seminal work. NETs possess a breadth of active vocabulary, can use appropriate idiom, harness intuition about usage and provide an insider's cultural knowledge (Barratt \& Kontra, 2000) of a target language community. They provide a need for students to engage in authentic 
English use (Barratt \& Kontra, 2000), may be less reliant on textbooks as teaching aids, bring different perspectives to materials and thus have some novelty value, at least initially. These strengths are, in general, the relative weaknesses of LETs whose own strengths, on the other hand, reflect the relative weaknesses of NETs. Medgyes (1994) argues that LETs can be positive role models for students; are generally better placed to anticipate language difficulties; and make profitable use of the mother tongue, with consequent richer resources for explaining some grammatical points. In addition, they are likely to have better familiarity with local syllabuses and examinations (Tang, 1997) and may often find it easier to develop close relationships with students. In the final analysis neither NET nor LET is inherently superior to the other (Medgyes, 1992). Policymakers may regard team teaching between NETs and LETs as a useful strategy to harness respective strengths and minimise weaknesses.

Team teaching is an important area of collaboration between NETs and LETs. For the purposes of this paper, we define team teaching as simply two teachers together in the classroom, actively involved in instruction. There is a body of literature (e.g. Thomas, 1992; Villa et al., 2004) related to team teaching as a general phenomenon in both school and university contexts. The main advantages for students of team teaching are as follows (adapted from Buckley, 2000): there are more opportunities for individual or small group support because two teachers are present in the classroom; the presence of different personalities, teaching styles and voices can make the classroom more lively and students can choose to interact more with whichever co-teacher they relate to better.

With respect to team teaching partnerships, we found most useful George and Davis-Wiley's (2000) reinforcement of earlier studies suggesting three essential characteristics of good partnerships: the mutual satisfaction of selfinterest or getting something from the partnership; a measure of selflessness on the part of each partner, a willingness to compromise or make some sacrifices for the benefit of team harmony; and dissimilarity between the partners so that they can complement each other. The last point is, however, debatable because some writers (e.g. Murata, 2002) adopt the perspective that while complementary talents are desirable, there should be shared philosophies and compatible professional attitudes between team teaching partners.

Much of the existing literature (e.g. Boyle, 1997; Lai, 1999) and more informal media reports have focused mainly on problematic issues in NET schemes in Hong Kong. Whilst acknowledging these exist, our aim in this paper is to discuss cases of good practices between NETs and LETs. We believe that discussing examples of successful collaboration can serve as a springboard for further development both in Hong Kong and elsewhere. With that aim in mind, our study is exploratory and focuses on the potential of team teaching and its impacts on students and teachers. In keeping with an exploratory study, our claims are necessarily tentative and clearly carry limitations in terms of generalisability. Through the study, we do hope to provide a stimulus for further consideration of collaborative English language teaching in schools and it is suggested that the paper will be of particular interest to colleagues, both practitioners and policymakers, in countries where some form of native/non-native team teaching is found. 


\section{Previous Findings on Collaboration in NET Schemes}

Here we review relevant literature related to NET schemes in Hong Kong, and team teaching or collaboration between NETs and LETs both in Hong Kong and elsewhere. In the Hong Kong context, Storey et al. (2001) found that at the secondary level there was a lack of genuine collaboration between NETs and LETs in that usually each worked in their own classroom with little sharing and understanding of what their counterparts were doing. Storey et al. reported little evidence of team teaching between NETs and local teachers or what they perceived as non-optimal forms of team teaching, for example, a LET doing simultaneous translation into the mother tongue with a low ability class.

Storey et al. (2001) found evidence that lower ability students' English improved more when they were taught by a combination of NET and LET rather than one of these on their own. The NETs themselves also reported difficulties when carrying out sole teaching with low ability students who lacked motivation to learn through English. Such findings reinforced an earlier study (Johnson \& Tang, 1993) which found NETs faced discipline problems with less academically oriented and/or less motivated students, an inability to use the students' mother tongue being particularly disadvantageous.

In terms of teaching approaches, Storey et al. also found that there was little shared understanding or common philosophy between LETs and NETs. They reported that local teachers set more homework, were more textbook-based, tended to correct students as soon as they made an error and used the mother tongue more frequently. ${ }^{3}$ Lack of shared philosophies could be exacerbated when NETs were seen as a threat to the self-esteem of LETs (Boyle, 1997), an issue intensified when NETs were perceived as too critical of local practices (Johnson \& Tang, 1993). Storey et al. (2001) concluded that the effects of NETs are unlikely to be significant without a culture shift involving an orientation towards more open collaboration between teachers and less emphasis on a culture of textbooks and exam preparation.

In Hong Kong, NETs are trained and experienced teachers, although they may not have much experience or any training in collaborative forms of teaching. Other schemes in the Asia Pacific region or Eastern Europe more explicitly emphasise collaboration, but often with untrained teachers. The Japan Exchange and Teaching programme (JET) employs mainly young unqualified native-speaking graduates to carry out team teaching with Japanese teachers of English (Gorsuch, 2002; McConnell, 2000). Tajino and Tajino (2000) argue that NETs $^{4}$ and LETs in Japan should have their distinctive roles in the classroom and what is important is team learning, whereby teachers as well as students are encouraged to be open-minded in learning from each other. They acknowledge that this was rarely the case and much of the literature reports challenges with unclear roles and lack of training or experience in collaborative forms of teaching being particular problems (Gorsuch, 2001; Mahoney, 2004).

With respect to South Korea, the English Programme in Korea (EPIK) scheme was based on JET (Kwon, 2000) and has been criticised for its failure to engender successful co-operation between NETs and LETs, with the catch-all of 'cultural differences' being identified as the main culprit (Choi, 2001). Overall, more conflict than collaboration has been reported, with a particular problem being 
a failure to integrate a team taught lesson with other elements of an essentially examination-driven curriculum. A consequence is that NETs have been mainly withdrawn from team teaching in schools in favour of positions as language instructors in teacher training institutes.

Alderson et al. (2001) report on the deployment of unqualified and inexperienced language assistants in Slovenian schools. The presence of language assistants was seen as beneficial to students and LETs, particularly in raising intercultural awareness. It was found that LETs tended to observe the language assistants conducting the lessons rather than to cooperate actively in the teaching process. For their part, the NET assistants preferred the freedom and independence of sole teaching rather than team teaching.

In sum, this literature does not report many positive examples of collaboration between NETs and LETs, providing an impetus for the current study. The relative dearth of successful experiences underpinned our wish to identify and disseminate good practices.

\section{Case Study Method}

The Storey et al. (2001) research, cited extensively above, was a comprehensive study carried out according to standard sampling techniques. We wished to extend this work by more fine-grained case studies, focusing on good practices. Our cases are outliers, NETs in partnership with LETs who were able to instigate positive forms of collaboration in their schools. The justification for the case studies is that we need examples of successful practices in order to provide a basis for ongoing improvement of collaboration between NETs and LETs. Learning from cases is an important means of development. Thus, these particular cases represent 'illuminatory instances' (Holliday, 2002) whose purpose is to exemplify the experience of two groups of teachers.

Our choice of cases arose from NETs viewed by the school community as good collaborators. The cases are Anna, a highly experienced language arts teacher and teacher adviser from Australia; and Pat, an Irishman, a qualified counsellor and foreign language major, who had previously worked for three years on the JET scheme. Anna was approached as a research participant because her collaborative work had been publicised in a good practices publication (Education Department, 2000), while Pat was approached because he had been described by his panel chair ${ }^{5}$ as 'the NET from paradise'. Based on Hong Kong norms, Pat's students were of above average academic ability, while Anna's were somewhat below average. Team teaching in Hong Kong secondary schools is rare (Storey et al., 2001) and that both Anna and Pat had been team teaching in their schools for four years was indicative of their special qualities which we believed could illuminate collaborative practice. Further contextual background $^{6}$ is not provided for reasons of space and because our intentions are to raise issues for further exploration rather than to make universal claims.

The main means of data collection was through interviews and classroom observations, the latter supported by detailed field notes. Semi-structured interviews were conducted with the NETs themselves and their LET co-teachers. The two NETs were tracked for one school day on two occasions several weeks apart. Indepth interviews with the NETs occurred at two points during each day in addition 
to less formal conversations immediately before and after observed lessons. Interviews were also carried out with five co-teachers in the two schools, as well as with the two school principals. Informal discussions were held with students.

Classroom observations were carried out in order to gauge how teachers were engaging with students, and understand the forms and impacts of various methods of collaboration. A total of four double lessons of 70 minutes each were observed for each NET. All the observed lessons were team taught. Detailed field notes were collected consisting of chronological descriptions of teacher and student behaviour during lessons and teacher and student talk recorded verbatim, with evaluative comments and issues for clarification to be followed up in interviews. Although having limitations, we felt that these procedures provided sufficient data for the purposes of an exploratory study. Standard qualitative data analysis procedures were used, involving coding and categorising data related to the focus of the research.

\section{Findings and Discussion}

The following section presents the findings from the two case studies. We address three main categories: nature of collaboration; impact on students; and impact on teachers.

\section{Nature of collaboration}

Pat and Anna taught their own classes as sole teachers and also team-taught 10 lessons per week with lower secondary classes (Years 7/8/9). Pat's lessons were all team-taught in classes of around 40 . Sometimes he introduced a unit and sometimes followed up the LET's input (e.g. Extract 1 below). Anna, in contrast, usually gave 'introduction to unit' lessons as sole teacher, via computer-based presentations in the school hall to 5-6 classes totalling around 150-200 students en masse. Although highly unusual, she found this form of mass presentation to be resource-efficient and facilitated provision of time for other more focused follow-up elements. This was Anna's preferred method, after four years of experimenting, as she felt her mass presentations, made more accessible by visuals, offered richer content and vocabulary than the textbook, thereby bringing the topic alive to students and the collaborating LETs present. For these solo presentations, Anna was supported by the regular LET teachers who would help to ensure students were on track and could also assist her preparation, for example when it was felt that a Chinese glossary was needed for key expressions. The presentation was followed by tailored, oralfocused collaborative lessons to individual classes.

In both case study schools, team teaching was generally executed with the NET taking the leading role, the LET supporting, and oral work predominating. An illustration of team teaching in practice is shown in Extract 1 from one of Pat's lessons with a Year 7 class of 38 students on the textbook topic of 'shopping tourism'.

Extract 1 illustrates a common form of NET/LET role delineation that we observed, with the NET taking the lead and the LET supporting, whilst together they demonstrated dialogues or modelled interaction, with both teachers available to support students during activities. In sum, the particular advantages of team teaching in this case were that the two teachers can demonstrate 


\section{Extract 1}

- Short NET \& LET interaction about not going shopping and planning other activities instead, such as sightseeing.

- NET gives out a worksheet and instructions for the first task: students look at a map and decide eight places to see and what tourists could do there.

- Students work in groups of 4-6 with coloured maps spread out amongst them. They speak in Chinese and in English. Teachers move among groups. Some students speak to the LET in Chinese and at one point a student speaks animatedly with the NET in English, trying to understand what the NET is saying. The LET comes to assist.

- NET and LET model a prepared dialogue as input for a mini-role-play task:

NET: Excuse me, do you know any interesting places to go in Hong Kong?

LET: Lan Kwai Fong.

NET: If I go to Lan Kwai Fong, what can I do?

LET: You can drink, eat and meet different people.

- NET then asks some individual students to take up his role in the dialogue and practise the task with him for the whole class. He says to them 'If I can read your name in Chinese you have to come out to do the role play, okay?' The class seems to enjoy listening to his attempts at Chinese pronunciation.

- Two students demonstrate the dialogue at the front of the class.

- NET instructs students to leave their seats and do the mini-role-play with 'at least three other classmates', marking the interlocutor's 'interesting place' on a sheet provided. Students participate willingly and do the roleplay with numerous partners, with some interacting with more than a dozen classmates. The majority are observed to speak enthusiastically without reading any written cues. Both NET and LET also take part in the activity. Some corrective feedback is heard from both teachers, but they appear to encourage participation more than accuracy.

dialogues; they are available to support students; and they can monitor or facilitate group activities in a large class.

One of the particular contributions of the LET in this kind of NET-led lesson was to harness the students' first language for English-learning purposes or facilitate the smooth flow of the lesson. For example, we also observed LETs: briefly introducing the lesson's aims in Cantonese with weaker students; checking that students could supply Chinese translations for new words; delivering complex instructions in the mother tongue about the criteria for choosing suitable students for role plays; and working with weaker or less confident students during group work. This exploitation of the mother tongue was something that the NET could not adequately do without the support of the LET. 


\section{Impact on students}

We now turn to the data which illuminates the impact on students as second language learners. We believe that the case studies illustrate that LETs agreeing to co-teach with a NET, resulted in somewhat atypical but beneficial learning experiences for students in that the NETs relative strength in lexical knowledge and fluency in speech could be exploited to further enrich the learning opportunities provided by the LET.

A feature of these collaborative lessons particularly significant to us as experienced observers of LETs teaching alone in similar classes, was the amount of extended speech, often containing complex clauses. An example (Extract 2) of extended speech ${ }^{7}$ modelled by the NET for student uptake occurred in Year 8 in a discussion of favourite countries:

\section{Extract 2}

NET: While I am there [Switzerland], I would taste the beautiful sweet chocolate, because in Switzerland they make the most beautiful chocolate in the world. I also want to go hiking, because in Switzerland they have very high mountains. I would buy a present for Miss $\mathrm{C}$ because she likes to eat good chocolate.

This is a long model for a below average Year 8 class, but it seemed to be understood and was taken up by some students, as shown in the complex clauses in Extract 3 attempted in students' pair-work.

\section{Extract 3}

Student A: I like to go to the [sic] England because I like to go there to play football and my parents are there...

Student B: I'd like to go to Japan because it's very romantic.

Two other students, who received focused support from both NET and LET, produced responses of four sentences, probably the longest unscripted turns we had heard from Year 8 students in Hong Kong. Support for our interpretation emerged in interviews, e.g. LETs commented that Anna's expectations of what students could do in English were higher than theirs. One LET observed, 'I [now] know better what students could do ... I didn't think that students can understand or say those things, but they do!' Another stated, 'She packs a lot of things into her lessons ... she's demanding'. In other words, we interpret team teaching as permitting teachers to discover student abilities (or problems) that they might not identify when teaching solo.

We also perceived that learning in these collaborative classes was more motivating than in a LET or NET solo class for several reasons. Firstly, at a basic perceptual level, there was more varied input in that there were two voices, two accents, two speeds of speech delivery. Secondly, there was relatively higher situational authenticity in the NET/LET interaction than in the somewhat 
stilted textbook dialogues often used as input in second language classes in Hong Kong. The more spontaneous interaction is thought to be more engaging (Kramsch \& Sullivan, 1996) partly because the two teachers were able to impart an element of liveliness and humour in tone and facial expressions. Extract 4 illustrates this point for Pat's Year 8 class:

\section{Extract 4}

NET: (chatty tone, slightly comic stance): Do you like shopping, Mr Y?

Mr A: (looking undecided): Not much.

NET: $\quad$ Me neither. I HATE shopping (screwing up nose, turning down mouth), so ... (to the class) if you don't like shopping (pause) what can you do in Hong Kong? (gesturing in appeal to the class).

Student: [If] You don't like shopping you can ... you can go inside and see something with four eyes (student is referring to one of the strange exhibits at 'Ripley's Believe it or Not', located at a popular tourist site).

There were numerous other examples of such 'public' interaction between NET and LET which we interpret as having the potential to motivate students towards learning inasmuch as the LET operates as a role model for them as potential interlocutors with other English users. The relatively authentic (in the sense of relating to a communicative need) NET-LET interaction makes it salient to students that English is a real language of communication, not just something needed for examination purposes.

Another reason why we believed that the team taught collaborative classes were more motivating than non-collaborative classes was because they appeared to involve higher levels of student intellectual engagement. This may have been due to the way in which the strengths of NET and LET were consciously exploited. In a class with limited English, a motivating level of cognitive activity might not be possible for a teacher, such as a NET, who was a non-speaker of the students' L1. Conversely, some open-ended activities (especially with older or higher ability students) might put strain on the linguistic resources of the LET. One of Anna's strategies for maintaining a motivating intellectual level with such students was to use her collaborating partner's facility in the students' L1. For example, when eliciting free responses she instructed a below average Year 8 class to 'tell Ms L in Chinese' and then, together with the LET, negotiated an appropriate version of the students' words in English on the board. In this way, students were supported to take more topic control (Ellis, 1999) and thus to communicate at a cognitive level relatively closer to their L1 ability, making meanings with more personal investment. The NET could not carry this out solo unless speaking good Cantonese and if the LET were to do this on her own, she would risk losing face if unable to translate fluently off the cuff from Cantonese to English.

Another factor which seemed to increase motivation was that the presence of two teachers provided a higher degree of support for students and hence a better opportunity for successful task completion. For example, after the NETs' content presentation, NET and LET together supported students in individual 
or group work. The extra attention available to students in team taught classes appeared to greatly facilitate on-task behaviour and we perceived that tasks were completed more effectively than in other observations of solo lessons which our roles as in-service teacher educators have involved us.

\section{Impact on teachers}

LET interviewees generally stated that they had benefited professionally from the collaboration with NETs, and that it had been a worthwhile experience. Some LETs mentioned they had developed aspects of English proficiency, e.g. quick repartee, use of slang, and wider vocabulary. Others mentioned expanding their teaching repertoire, for example using collaborative dictations; more effective use of pre-tasks; and integrating grammar into communicative activities:

'[Before] I asked students to just take dictation of a few items, but now they take dictation in groups and give the best answer as a group effort.'

'I used to just hand out a piece of paper with a task on it and give one or two points, and then ask the children to do it, [but I found Anna] broke up the task into small systematic steps ... models some acting out ... allows students to rehearse.'

'I try to be more dramatic and to integrate grammar with communication.'

A further illustration of NET impact on colleagues was exemplified by a LET expressing appreciation of the way Pat provided co-teachers with authentic internet resources for units they were team teaching. The LET regarded this as a time-saver so that he could spend more time on composition marking, a particular priority for LETs in Hong Kong (Davison, 2004). Collaboration did, however, also involve some sacrifices. A LET in Anna's school observed that the collaboration resulted in extra workload for her - '[in team teaching] I sometimes have to sacrifice things I need to do, but then I just do it [cover the textbook content] after school [in extra classes].'

Turning to the impact on NETs, their main professional development related to developing a deeper understanding of Chinese students' learning orientations and difficulties in studying English. They also became increasingly familiar with the nature of the local educational context. In addition, they learnt new things about Chinese language and culture, treasuring learning in this way from students and colleagues. Pat was particularly serious about making progress in both speech and writing in Chinese. One LET found this attitude 'valuable because students see the NET struggling with a second language as well'.

These NETs tried to fit into their school's culture and not risk alienating their colleagues by emphasising their English language superiority. For example, Pat said he deliberately 'let teacher language errors go in pursuit of a higher goal', in other words he was more interested in getting on with LETs than improving their English. Both NETs evidenced an ethos of encouragement and claimed to prioritise promoting the confidence of all English users they encountered. Both Anna and Pat were sensitive to the fact that LETs' language proficiency was often criticised by bodies such as government and media (Glenwright, 2005).

Whilst we judged the interactions between NETs and LETs to be largely 
positive, there were beneath the surface some tensions relating to differing views of suitable educational practices. Both NETs were aware of the need not to rock the boat too much, but at the same time, they wanted to retain their own professional identity. They emphasised that they found it vital to insist on 'different ways of being and thinking'. Pat's principal confirmed that 'he asks NOT to be treated like a local teacher'. An example related to Anna's firm commitment to language arts which was not mirrored by her LET counterparts. Anna's student-created drama performances and the external award-winning verse-speaking team remained part of Anna's extra-curricular responsibilities, peripheral to the main grammar-based curriculum.

A further example of differing practices and conceptions relates to assessment and marking. Both NETs found that LETs tended to spend term-time marking and testing excessively, rather than refining their teaching to enhance learning outcomes. Anna had jokingly but firmly made it clear she would not participate in activities which made the teacher appear hard-working, but which she saw as intellectually demeaning and educationally unsound, such as mechanically marking by 'ticking and flicking' the pages of large quantities of student exercise books. In this sense she was going against the prevailing assessment culture in Hong Kong (cf. Davison, 2004) and providing a challenge to the existing status quo. However, both NETs were aware that to compensate for their difference in teaching philosophies, they must be and be seen to be hard-working, in a culture where diligence is one of the most valued attributes (Cheng \& Wong, 1996). Thus, Pat's colleagues were aware of his spending lunchtimes conversing with students; and Anna's negotiated exemptions from meetings held in Cantonese were tolerated by her colleagues because of her additional duties in arranging extra-curricular activities such as annual overseas study tours, local excursions and weekend inter-school debates.

Despite differences in educational philosophies, the NETs in these schools were able to work collaboratively with LETs and saw it as important to show that they were willing to compromise and accept (though not necessarily agree with) some practices.

\section{Summary and Wider Implications}

In this section, we would like to draw together some themes from the study and also make some wider speculations which carry resonance beyond the Hong Kong context. We draw on the current study, our own previous experiences as non-native foreign language teachers, our roles as NETs in the late 1980s, our current positions as teacher educators working with LETs, our reading of the wider literature and our own other NET-related research (Carless, 2006; Walker, 2001). The discussion below is first related to our earlier framework of team teaching, then to the theme of differing educational philosophies and finally to more general points related to second language acquisition (SLA).

\section{Team teaching}

Unlike much of the reported findings for JET, EPIK and the project in Slovenia, we found that the teachers in the two schools were willing to collaborate. The NETs and LETs in our cases largely exhibited the strengths rather than the weak- 
nesses referred to in the introduction. They seemed to have developed ways of working which tended to maximise NET/LET respective strengths associated with observed positive student response. Thus, we believe that collaborations such as NET/LET do have a role to play as long as there are sufficient sensitivities, both cultural and interpersonal, and each party is able to show some respect and actual accommodation for the views and actions of their counterparts. NETs should not be too critical, but also need to maintain their own principles and support gradual changes in partnership with LETs.

We identified evidence that supported George and Davis-Wiley's suggestions for good collaboration involving mutual satisfaction of self-interest, willingness to compromise and complementarity. That is, firstly, there was evidence of mutual satisfaction of self-interest in that: several of our LET respondents felt they were experiencing increased opportunities to communicate in English and were being exposed to some useful teaching strategies; Pat was pleased to be learning some Chinese and building good relationships with students; Anna was satisfied that she could contribute to language arts and extra curricular activities while still teaching regular classes. Secondly, there was also plentiful evidence of selflessness of both NETs and LETs. For example, a LET gave up class time to the NET even though hard-pressed to complete the assigned textbook; LETs helped to translate into Cantonese for NETs; NETs tried to fit in with local and school educational cultures; and NETs were sensitive in not causing loss of face by correcting LETs' English inaccuracies. Thirdly, there was evidence of complementarity of their preferred approaches. For example, the more communicative fluency-focused nature of the NET-led lessons seemed to be balanced by focus on form in LET solo lessons. We have also seen that NETs negotiated to be treated differently from LETs, such as Anna's organisation of extra-curricular activities instead of attending meetings and that NETs consciously behaved differently from LETs as teachers in focusing on oral English, using non-textbook, non-test-oriented materials, or providing cognitively rich learning opportunities.

We believe that diversity is an asset in team teaching as it enables partners to showcase different talents and emphasise different elements of the teaching and learning process. This diversity does need to be balanced by some form of empathy for the views of the partner, even when they may be sharply different.

\section{Reconciling differing educational philosophies}

Following from the previous section, we believe that one of the key factors in successful collaboration is reconciling or accepting differing educational philosophies. In other words, acknowledging that one is different but accepting that partners have their own beliefs, culture and reasoning; or to put it succinctly, 'to agree to differ'. These differences may well occur with team teachers from the same culture, but are even more likely to arise with teachers from different countries. Collaborating NETs need to tread a fine line between accepting local school cultures and jeopardising smooth relationships by critiquing current practices.

Our cases provided evidence of gaps in conceptions of how students learn and differences in professional role perceptions, and this is reaffirmed 
elsewhere (e.g. Mahoney, 2004). Our interpretation of the wider literature (e.g. McConnell, 2000; Moote, 2003; Reves \& Medgyes, 1994) is that NETs tend to see LET teaching as focused too much on the textbook, prioritising grammar over fluency and involving excessive testing. LETs, in contrast, often find that NETled teaching does not always result in solid learning of the things they value, such as textbook content and assessed grammar conventions. NET lessons (and this is particularly the case in the JET scheme [McConnell, 2000]) may remain somewhat peripheral when oral-based input is seen as running counter to LET priorities of grammar, textbook completion or test preparation.

Within schemes involving NETs and LETs from contrasting backgrounds, differences are inevitable but the negative impacts can be minimised when both collaborators are: well-trained in ELT; more experienced or more capable in collaboration; more culturally or interpersonally sensitive; and more positive towards collaboration. Such team teaching will also have greater impact when team taught lessons are better integrated with other school, teacher or student priorities, e.g. passing exams. Administrators can support these processes by providing scheduled opportunities for professional exchange and staff development for enhancing capacities of both NETs and LETs.

\section{Implications for SLA}

We turn finally to the issue of the relationship between collaboration and SLA. We believe that the NET / LET team teaching documented here had potential for positive impacts on students' SLA in terms of the possible enrichment of two of the conditions for learning espoused in the SLA literature; namely comprehended input (Ellis, 1999) with 'pushed' output opportunities (Swain, 1995), and motivation (Dornyei \& Schmidt, 2001).

NET/LET oral interaction is a rich source of input for students. As visible, 'modified' interaction, exemplified by our data, it is likely to be more frequent, better comprehended and more processable by students (Ellis, 1999) than, for example, recorded interactions. Furthermore, with LET immediate mediation, NETs' monologic input, in our cases deliberately semantically and grammatically more elaborated, could provide appropriate noticing opportunities (Skehan, 1998), be more immediately part of 'uptake' (Allwright, 1984) and more directly constructed or lexicalised by students (Skehan, 1998). With respect to the use of the mother tongue during team taught lessons, Lin (2000) provides evidence of judicious use of first language as a resource for obtaining input in a second, especially with less able learners. Use of the mother tongue can also play a role in reducing anxiety and creating a comfortable classroom atmosphere.

In terms of the 'pushed' output associated with comprehended input, we have seen that NET / LET cooperation, especially during whole class eliciting allowed spontaneous, accurate feedback to students to use L2 resources not yet fully under the students' control. This raised the L2 output quality to approximate the students' L1 competence. The qualitatively pushed output arose through LET-mediated negotiation involving the use of repetition, expansion, extension and prompting of the students' output by both teachers, all of which in turn act as input to listeners. In addition, NET and LET together could clearly push output in terms of quantity, especially during individual work or group activities. Together they doubled the space for students to speak to the teacher for a 
longer time, more frequently, and / or use more words. This greater quantitative extendedness may in the longer term enhance automaticity and fluency.

Regarding motivation, we believe that NET/LET co-teaching potentially provides more motivating language learning experiences, in line with McConnell (2000), who argues that in the JET scheme co-taught lessons had a positive impact on student motivation. At a basic perceptual level, as mentioned, NET/ LET voice variation can raise students' attention to input, potentially increasing chances of successful output and hence future expectations of success. In terms of task value, students engage with tasks involving intellectual challenge beyond the purely grammatical level (Ellis, 1999). We have seen this prioritised by the NETs. However, it was smoothly facilitated through LET expertise, especially with weaker or less manageable students. That is, weaker students, encouraged to respond by the more engaging topics may even take topic control where they are confident the LET could support gaps in their English. Students' being motivated enough to take topic control is theorised to have positive implications for 'uptake' (Ellis, 1994), as other students may be more likely to take up what has been topicalised by peers than by teachers.

In sum, NET / LET collaborative teaching can be a valuable way of enhancing SLA opportunities provided in sole teacher classes, especially where NET/LET capacities are sufficiently different but compatible, and especially for secondary school students with relatively low English proficiency.

\section{Conclusion}

This paper has discussed team teaching in a specific context. We believe that collaboration between NETs and LETs is a challenging task but when handled thoughtfully can showcase the strengths of each party and minimise their weaknesses. As a final word, we would like to reiterate our call for more reporting of good practices of collaboration between NETs and LETs, which we believe can provide potential for ongoing improvement of team teaching.

\section{Correspondence}

Any correspondence should be directed to Dr David Carless, Faculty of Education, University of Hong Kong, Hong Kong, China (dcarless@hku.hk).

\section{Notes}

1. Native and non-native speakers are used as descriptive terms, following the dominant discourse. We acknowledge that these terms are not ideal and have been extensively critiqued in the literature (e.g. Cook, 1999; Davies, 2003).

2. Since 2002, NET schemes have been expanded into the primary sector, see Carless (2006) for a discussion.

3. Similar findings are reported in a study focusing on NET and LET teaching behaviours in ten countries (Reves \& Medgyes, 1994).

4. The actual terms used in the JET scheme are Assistant Language Teachers (ALTs) and Japanese Teachers of English (JTEs).

5. The role of panel chair is roughly akin to a departmental coordinator or head of department.

6. Recent discussions of the culture of the Hong Kong secondary school classroom include Tsui (2003); Lin and Luk (2002).

7. Our experience leads us to believe that it would be rare for a LET in Hong Kong to produce such a lengthy non-textbook related utterance. 


\section{References}

Alderson, J.C., Pizorn, K., Zemva, N. and Beaver, L. (2001) The Language Assistant Scheme in Slovenia: A Baseline Study. Ljubljana: Ministry of Education, Science and Sport.

Allwright, D. (1984) Why don't learners learn what teachers teach? - The interaction hypothesis. In D. Singleton and D. Little (eds) Language Learning in Formal and Informal Contexts. Dublin: IRAAL.

Barratt, L. and Kontra, E. (2000) Native English speaking teachers in cultures other than their own. TESOL Journal 9 (3), 19-23.

Boyle, J. (1997) Native speaker teachers of English in Hong Kong. Language and Education 11 (3), 163-81.

Buckley, F. (2000) Team Teaching: What, Why and How? Thousand Oaks, CA: Sage.

Carless, D. (2006) Collaborative EFL teaching in primary schools. English Language Teaching Journal 60 (4).

Cheng, K.M. and Wong, K.C. (1996) School effectiveness in East Asia: Concepts, origins and implications. Journal of Educational Administration 34 (5), 32-49.

Choi, Y. (2001) Suggestions for the re-organisation of English teaching program by native speakers in Korea. English Teaching 56, 101-22.

Cook, V. (1999) Going beyond the native speaker in language teaching. TESOL Quarterly 33 (2), 185-209.

Davies, A. (2003) The Native Speaker: Myth and Reality (2nd edn). Clevedon: Multilingual Matters.

Davison, C. (2004) The contradictory culture of teacher-based assessment: ESL teacher assessment practices in Australian and Hong Kong secondary schools. Language Testing 21 (3), 305-34.

Dornyei, Z. and Schmidt, R. (eds) (2001) Motivation and Second Language Acquisition. Honolulu, Hawaii: Second Language Teaching and Curriculum Centre, University of Hawaii.

Education Department (2000) NET-working: Examples of Good Professional Practice within the NET Scheme. Hong Kong: Education Department.

Ellis, R. (1994) The Study of Second Language Acquisition. Oxford and New York: Oxford University Press.

Ellis, R. (1999) Learning a Second Language through Interaction. Amsterdam: John Benjamins.

George, M. and Davis-Wiley, P. (2000) Team teaching a graduate course. College Teaching 48 (2), 75-80.

Glenwright, P. (2005) Grammar error strike hard: Language proficiency testing of Hong Kong teachers and the four 'Noes'. Journal of Language, Identity and Education 4 (3), 201-26.

Gorsuch, G. (2001) Japanese EFL teachers' perceptions of communicative, audiolingual and Yakudoku activities: The plan versus the reality. Education Policy Analysis Archives 9 (10).

Gorsuch, G. (2002) Assistant foreign language teachers in Japanese high schools: Focus on the hosting of Japanese teachers. JALT Journal 24 (1), 5-32.

Holliday, A. (2002) Doing and Writing Qualitative Research. London: Sage.

Johnson, K. and Tang, G. (1993) Engineering a shift to English in Hong Kong schools. In T. Boswood, R. Hoffman and P. Tung (eds) Perspectives on English for Professional Communication. Hong Kong: City Polytechnic of Hong Kong.

Kramsch, C. and Sullivan, P. (1996) Appropriate pedagogy. ELT Journal 50, 199-212.

Kwon, O. (2000) Korea's English education policy changes in the 1990s: Innovations to gear the nation in the 21st century. English Teaching 55, 47-92.

Lai, M.L. (1999) JET and NET: A comparison of native speaking English teacher schemes in Japan and Hong Kong. Language, Culture and Curriculum 12 (3), 215-28.

Lin, M.Y.A. (2000) Deconstructing 'mixed code'. In D.S.C. Li, A. Lin and W.K. Tsang (eds) Language and Education in Postcolonial Hong Kong (pp. 179-94). Hong Kong: Linguistic Society of Hong Kong.

Lin, M.Y.A. and Luk, J. (2002) Beyond progressive liberalism and cultural relativism: Towards critical postmodernist, sociohistorically situated perspectives in classroom studies. Canadian Modern Language Review 59, 97-124. 
Mahoney, S. (2004) Role controversy among team teachers in the JET programme. JALT $26(2), 223-44$.

McConnell, D. (2000) Importing Diversity: Inside Japan's JET Program. Berkeley, CA: University of California Press.

Medgyes, P. (1992) Native or non-native: Who's worth more? English Language Teaching Journal 46 (4), 340-9.

Medgyes, P. (1994) The Non-native Teacher. London: MacMillan.

Moote, S. (2003) Insights into team teaching. English Teacher 6 (3), 328-34.

Murata, R. (2002) What does team teaching mean? A case study of interdisciplinary teaming. Journal of Educational Research 96 (2), 67-77.

Reves, T. and Medgyes, P. (1994) The non-native English speaking EFL/ESL teachers' self-image: An international survey. System 22 (3), 353-67.

Skehan, P. (1998) A Cognitive Approach to Language Learning. Oxford: Oxford University Press.

Storey, P., Luk, J., Gray, J., Wang-Kho, E., Lin, A. and Berry, R.S.Y. (2001) Monitoring and evaluation of the native-speaking English teacher scheme. Unpublished research report: Hong Kong Institute of Education.

Swain, M. (1995) Three functions of output in second language learning. In G. Cook and B. Seidlhofer (eds) For H.G. Widdowson: Principles and Practice in the Study of Language. Oxford: Oxford University Press.

Tajino, A. and Tajino, Y. (2000) Native and non-native: What can they offer? Lessons from team-teaching in Japan. ELT Journal 54 (1), 3-11.

Tang, C. (1997) On the power and status of non-native ESL teachers. TESOL Quarterly 31 (3), 577-80.

Thomas, G. (1992) Effective Classroom Teamwork: Support or Intrusion? London and New York: Routledge.

Tsui, A.B.M. (2003) Understanding Expertise in Teaching: Case Studies of ESL Teachers. Cambridge: Cambridge University Press.

Villa, R.A., Thousand, J.S. and Nevin, A.I. (2004) A Guide to Co-teaching: Practical Tips for Facilitating Student Learning. Thousand Oaks, CA: Corwin.

Walker, E. (2001) Roles of native-speaker English teachers in Hong Kong secondary schools. Asia Pacific Journal of Language in Education 4 (2), 51-77. 